\title{
Vivências de familiares frente à situação de hospitalização
} Eliza Maria Rezende Dázio, D.Sc.*, Carlos Augusto Mariotto Alves**, Sara Rodrigues Rosado, M.Sc.***,
Cibelle Barcelos Filipini****, Silvana Maria Coelho Leite Fava, D.Sc. ${ }^{* * * * *}$, Rogério Silva Lima, M.Sc. ${ }^{* * * * *}$, Ana Mariele de Souza ${ }^{* * * * * *}$

*Enfermeira, Professora do Curso de Graduação e Pós-Graduação em Enfermagem da Universidade Federal de Alfenas (UNIFAL-MG), **Graduando em Enfermagem da UNIFAL-MG, ${ }^{* * *}$ Enfermeira responsável pelo Centro Municipal de Atenção às Pessoas com Deficiência da Prefeitura Municipal de Alfenas, ${ }^{* * *}$ Enfermeira, Mestranda em Enfermagem, Professora do Instituto Federal do Sul de Minas, ${ }^{* * * *}$ Enfermeira, Professora do Curso de Graduação e Pós-Graduação em Enfermagem da UNIFAL-MG, ${ }^{* * * * *}$ Enfermeiro, Professor da UNIFAL-MG, ${ }^{* * * * * *}$ Graduada em Enfermagem da UNIFAL-MG

\section{Resumo}

Objetivo: Identificar as evidências científicas disponíveis na literatura relacionadas às vivências de familiares/ acompanhantes frente à situação de hospitalização. Material e métodos: Revisão integrativa com vista a responder a questão: O que tem sido publicado sobre as vivências dos familiares frente à situação de hospitalização? Foi realizada busca de artigos publicados de 2009 a 2014, utilizando um instrumento de Ursi e nas bases de dados Lilacs, Medline, BDENF, IBECS e Scielo. Resultados: Sete estudos foram selecionados, publicados no período de 2009 a 2011, em periódicos nacionais, na língua portuguesa, que foram realizados em ambiente hospitalar em diferentes setores de internação. Foi possível observar a predominância da figura materna como acompanhante, mas também os pais foram incluídos nos estudos. Conclusão: A enfermagem deve estar preparada para atender às necessidades referidas pelos familiares, relacionadas à experiência com a internação, percebendo a família como foco e não como apêndice do cuidado.

Palavras-chaves: hospitalização, cuidadores, Enfermagem.

\section{Abstract \\ Experiences of families facing the situation of hospitalization: an integrative review}

Objective: To identify the scientific evidence available in the literature related to the experiences of family members/ caregivers about the situation of hospitalization. Method: Integrative review aimed to answer the question: What has been published about the experiences of family members about the situation of hospitalization? Search for articles published from 2009 to 2014 was performed using an instrument adapted from Ursi and in databases in Lilacs, Web of Science, Medline, IBECS, Pubmed, BDENF and Scielo. Results: Seven studies which were published from 2009 to 2011, in national journals, in Portuguese, and performed in hospitals in different sectors of hospitalization were selected. We may observe the predominance of the maternal figure as a companion, but also the parents were included in the studies. 
Conclusion: Nursing should be prepared to meet the needs reported by family members, in relation to their experience with the hospital, seeing the family as the center and not as a part of care.

Key-words: hospitalization, caregivers, Nursing.

\section{Resumen}

\section{Vivencias de miembros de la familia que enfrentan la situación de hospitalización}

Objetivo: Identificar la evidencia científica disponible en la literatura relacionada con las vivencias de miembros de la familia/cuidadores acerca de la situación de hospitalización. Método: Revisión integradora dirigida a responder a la pregunta: ¿Qué se ha publicado sobre las experiencias de los miembros de la familia sobre la situación de hospitalización? Búsqueda de artículos publicados desde 2009 hasta 2014 se llevó a cabo utilizando un instrumento adaptado de Ursi y en las bases de datos Lilacs, Medline, BDENF, IBECS y Scielo. Resultados: Siete estudios que fueron publicados en el período 2009-2011, en revistas nacionales, en portugués, y realizados en ambiente hospitalario en los diferentes sectores de hospitalización fueron seleccionados. Es posible observar el predominio de la figura materna como acompañante, pero también los padres fueron incluidos en los estudios. Conclusión: La enfermería debe estar preparada para responder a las necesidades relatadas por los miembros de la familia, con respecto a la experiencia hospitalaria, percibiendo a la familia como el centro y no como una parte de la atención.

Palabras-clave: hospitalización, cuidadores, Enfermería.

\section{Introdução}

O processo patológico e a hospitalização inauguram um fato novo para as famílias, impondo-lhes uma reestruturação de horários e afazeres, além de submetê-las a um ambiente estranho que fomenta sofrimento físico e emocional. Este fato faz com que os familiares se sintam esgotados e muitas vezes ignorados em suas necessidades, o que pode tornar a hospitalização uma experiência traumatizante [1].

A família é a primeira fonte de apoio, de cuidados e de auxílio a seus integrantes, uma vez que se encontra envolvida pelo vínculo da afetividade, oferecendo conforto e suporte emocional por meio de diálogos e conselhos, com o objetivo de diminuir o sofrimento, a dor e o medo [2].

A necessidade da presença de um membro da família é reforçada por vários estudos, ao considerarem que o ente adoecido é dependente de seus familiares e que a hospitalização o distancia de seu lar. A participação do familiar neste processo é essencial, pois a atividade de cuidar realizada com a equipe de saúde no hospital torna-o parceiro da equipe [3].

O apoio do familiar acompanhante durante a hospitalização, tão importante e necessário, foi assegurado pelo Ministério de Estado da Saúde, com intuito de garantir a melhoria da qualidade de vida do paciente, especialmente quando se trata de crianças e idosos. Além de tornar obrigatórios os meios que viabilizam a permanência do acompanhante, a Portaria No 280, de 7 de abril de 1999, do Ministério da Saúde, garante os recursos financeiros para sua acomodaçáo [4].

A importância da família no processo de hospitalização é uma questão incontestável, tanto para o paciente quanto para a equipe de enfermagem. No entanto, para um atendimento integral não podem ser desconsideradas suas necessidades e fragilidades, pois assim como o paciente, a família também precisa ser amparada afetivamente e sentir-se cuidada [5].

Incluir o familiar na assistência de enfermagem possibilita a instrumentalização dos profissionais para um melhor direcionamento de açóes e cuidados destinados à família e ao paciente hospitalizado. Ressalta-se, porém, que pouca ou nenhuma atenção tem sido oferecida ao familiar que exerce o cuidado, pois os profissionais de saúde acabam atendendo apenas a doença, esquecendo-se dos familiares que também necessitam de apoio, afinal serão eles que darão continuidade ao cuidado [6].

Neste contexto e diante da escassez de estudos sobre esta temática, decidimos realizar esta revisão integrativa com objetivo de identificar as evidências as evidências científicas disponíveis na literatura relacionadas às vivências de familiares frente à situação de hospitalização. 


\section{Material e métodos}

Trata-se se um estudo bibliográfico e documental efetuado por meio da revisão integrativa da literatura que objetiva agrupar e sintetizar resultados de estudos de um determinado tema, de maneira sistemática e ordenada a fim de contribuir para o aprofundamento do assunto delimitado [7] .

A pesquisa pautou-se pela seguinte questáo norteadora: $\mathrm{O}$ que tem sido publicado sobre as vivências dos familiares frente à situação de hospitalização?

Foram exploradas bases de dados eletrônicas da BVS: Literatura Latino-Americana e do Caribe através de Informações em Ciências da Saúde (Lilacs), Medline, BDENF, IBECS e a biblioteca virtual Scientific Electronic Library Online (Scielo). Para empreender a busca foram utilizados os Descritores em Ciências da Saúde (DeCS) associados em português "Hospitalização", "Familiares" e "Enfermagem", associados pelo boleano "and".

Adotou-se como critério de inclusão os artigos em Português, Espanhol e Inglês, disponíveis na íntegra, produzidos no período de 2009 a abril de 2014. Foram excluídas teses, dissertaçóes, livros, relatórios de conferências, resumos de congresso e artigos que não respondessem a questão norteadora.

Para a análise dos artigos selecionados utilizou-se um instrumento adaptado de Ursi [8] que favoreceu a identificação e organização dos seguintes elementos dos estudos: identificação do autor; características metodológicas; nível de evidência; resultados e conclusão, principais vivências relatadas pelos familiares frente à situação de hospitalização. Em seguida, os estudos foram classificados quanto ao nível de evidência, baseando-se na classificação de Melnyk e Fineout-Overholt [9].
A análise dos dados foi realizada em duas etapas, sendo a primeira constituída por síntese dos dados, identificação e caracterização da amostra, e a segunda etapa composta pela análise do conteúdo dos artigos.

\section{Resultados}

A busca resultou em 48 publicaçóes indexadas, entretanto, apenas 10 artigos atendiam aos critérios de inclusão. Dessas, foram submetidos à leitura do título e do resumo e observou-se que três (03) trabalhos não respondiam à questáo norteadora. Portanto, a amostra constituiu-se de sete (7) publicaçóes que foram analisadas detalhadamente e receberam um código com sequência alfanumérica (A1 a A7), para facilitar a identificação dos mesmos.

No que diz respeito aos sete artigos incluídos no presente estudo, estes foram publicados no período de 2009 a 2011, em periódicos nacionais, na língua portuguesa, sendo seis em revistas específicas da Enfermagem, conforme demonstrado na Tabela I.

Em relação à abordagem metodológica e método de investigação escolhidos, observou-se a predominância de estudos de natureza qualitativa. Dois trabalhos utilizaram como referencial teórico-metodológico a Teoria dos Sistemas de Betty Neuman; um trabalho utilizou a Teoria Fundamentada nos Dados; um fundamentou-se na abordagem da sociologia fenomenológica de Alfred Schutz e três não especificaram. Todos os artigos atenderam ao nível de evidência $\mathrm{V}$, conforme Tabela II.

A Tabela III apresenta uma síntese dos artigos incluídos neste estudo de revisão, destacando-se os principais resultados e conclusão.

Os estudos selecionados, em sua totalidade foram realizados no ambiente hospitalar em dife-

Tabela I - Distribuição dos artigos por autor, pais de estudo, periódico, ano e língua de publicação. Alfenas/MG, 2014.

\begin{tabular}{llllll}
\hline ID & \multicolumn{1}{c}{ Autor } & \multicolumn{1}{c}{ Periódico de publicação } & $\begin{array}{l}\text { País do } \\
\text { estudo }\end{array}$ & $\begin{array}{c}\text { Ano de } \\
\text { publicação }\end{array}$ & \multicolumn{1}{c}{ Idioma } \\
\hline A1 & Santos AMR et al. [10] & $\begin{array}{l}\text { Revista Escola de Enfermagem } \\
\text { da USP }\end{array}$ & Brasil & 2011 & Português \\
\hline A2 & Frizon G et al. [11] & Revista Gaúcha Enfermagem & Brasil & 2011 & Português \\
\hline A3 & Mello RM, Schneider JF [12] & Revista Gaúcha Enfermagem & Brasil & 2011 & Português \\
\hline A4 & Gomes GC et al. [13] & Revista de Enfermagem da UERJ & Brasil & 2011 & Português \\
\hline A5 & Vieira GB, Alvarez AM, Girondi JBR [14] & Revista Eletrônica de Enfermagem & Brasil & 2011 & Português \\
\hline A6 & Silveira RA, Oliveira ICS [15] & Revista RENE & Brasil & 2011 & Português \\
\hline A7 & Vieira GB, Alvarez AM, Gonçalves LTI [16] & Ciência, Cuidado e Saúde & Brasil & 2009 & Português \\
\hline
\end{tabular}


Tabela II - Distribuição dos artigos conforme abordagem metodológica e nível de evidência. Alfenas/MG, 2014.

\begin{tabular}{llc}
\hline ID & \multicolumn{1}{c}{ Tipo de Estudo } & $\begin{array}{c}\text { Nível de } \\
\text { Evidência }\end{array}$ \\
\hline A1 & Abordagem metodológica qualitativa. & $\mathrm{V}$ \\
\hline A2 & Abordagem metodológica qualitativa. & $\mathrm{V}$ \\
\hline A3 & $\begin{array}{l}\text { Abordagem metodológica qualitativa, método de investigação sociologia fenomenológica de } \\
\text { Alfred Schutz. }\end{array}$ & $\mathrm{V}$ \\
\hline A4 & $\begin{array}{l}\text { Abordagem metodológica qualitativa, método de investigação Teoria Fundamentada em Da- } \\
\text { dos. }\end{array}$ & $\mathrm{V}$ \\
\hline A5 & $\begin{array}{l}\text { Abordagem metodológica qualitativa, método de investigação Teoria dos Sistemas de Betty } \\
\text { Neuman. }\end{array}$ & $\mathrm{V}$ \\
\hline A6 & Abordagem metodológica qualitativa. & $\mathrm{V}$ \\
\hline A7 & $\begin{array}{l}\text { Abordagem metodológica qualitativa, método de investigação Teoria dos Sistemas de Betty } \\
\text { Neuman. }\end{array}$ \\
\hline
\end{tabular}

Tabela III - Síntese dos estudos selecionados nas bases de dados. Alfenas/MG, 2014.

ID Principais resultados Conclusões

$\begin{array}{lll}\text { A1 Verificou-se que são evidentes as mudanças que } & \text { O acompanhante passa por adaptações, ao vivenciar a } \\ \text { ocorrem e transformam todo o cotidiano familiar; } & \text { hospitalização, existindo alterações na rotina familiar. }\end{array}$ envolvendo alterações emocionais de todos os participantes do processo: pai/mãe e filho hospitalizado.

A2 A análise revelou sentimentos como: dor, angústia, Percebeu-se pelos relatos que um emaranhado de sentitristeza, impotência, medo, desespero, ansiedade e mentos acompanha os familiares durante a hospitalização expectativa infinita.

de seu parente na UTI e em especial no momento que Acredita-se que esses sentimentos seriam minimizados se o espaço da sala de espera fosse ocupado por multiprofissionais da saúde, em especial enfermeiros, que oferecessem o acolhimento a esses familiares, preparando-os, escutando-os e esclarecendo dúvidas.

A3 Ao internar o indivíduo em sofrimento psíquico, o familiar consegue ver a si mesmo, por ser esse um espaço que considera a individualidade de cada um, não apenas do indivíduo internado, mas também de seus familiares. antecede a visita.

Almeja-se que, no lado de fora e dentro da UTI, ocorram ações acolhedoras para os familiares, ajudando-os a enfrentar a hospitalização de um familiar na unidade crítica.

Torna-se fundamental que os profissionais de saúde e a sociedade exerçam sua crítica ao hospital como espaço restrito às doenças, trabalhando também a saúde do indivíduo em sofrimento psíquico e de sua família, tornando esse momento mais humano e aproximando a equipe das necessidades desses envolvidos (possibilitando que sejam agentes do cuidado).

A4 Observou-se que a família constrói uma rede social na qual recebe e dá apoio.

O apoio é manifestado no hospital através do recebimento de visitas; do núcleo familiar que se organiza para que Valoriza o apoio recebido de seus familiares, das outras famílias com quem convive, dos amigos, vizinhos e profissionais da saúde; se fortalece na esperança e na fé em Deus e constrói vínculos. este possa cuidar da criança no hospital e que assuma seu papel das atividades domésticas; dos profissionais da equipe de saúde; das outras famílias com quem convive no hospital; de amigos e vizinhos. A fé em Deus e a esperança na recuperação da criança apresentam-se como importantes potencializadores da família no hospital. 
A5 A perda da capacidade funcional é fator muito preocupante para os familiares, pois requer uma nova organização familiar para enfrentar a situação. A velhice não é sinônima de doença. Entretanto, em idades mais avançadas as limitações visuais, auditivas, motoras e intelectuais, bem como o surgimento de doenças crônico-degenerativas intensificam-se, ocasionando a dependência nas atividades cotidianas.

A6 Receber informação sobre o tipo de câncer e localização representa para os informantes (pais de crianças com câncer) segurança e conforto.

Os informantes relatam que no período de hospitalização e na ausência dessas informações é comum que o padrão de sono se torne irregular devido à preocupação com o estado da criança, ruído dos equipamentos, administração de medicamentos, e as poltronas de acompanhantes não oferecem conforto ou estão em má conservação.

O banho ocorre apenas durante o horário de sono da criança para que não fique agitada e sozinha.

O auxílio que recebem dos familiares, amigos, igreja e da comunidade, ocorre de forma espiritual através de palavras de conforto e demonstração de carinho e em outras vezes com apoio financeiro ou doações.

A7 Foram identificados como fator de estresse a preocupação constante dos cuidadores com a manipulação da sonda nasoenteral (SNE) e a realização de curativos ou procedimentos que exigiam habilidades específicas, principalmente se fosse necessária sua realização após a alta, no domicílio. Surgiram sentimentos como angústia devido à situação de dependência nos cuidadores, pois o ser humano tem como padrão de saúde a independência na realização das suas atividades diárias; ainda observa um sentimento de culpa e frustração em não poder oferecer à pessoa dependente o conforto merecido. Relações familiares conturbadas devido a problemas financeiros ou sobrecarga de alguns membros no cuidado ao idoso.
As informações obtidas demonstraram que as famílias se mobilizam para organizar o ato de cuidar de um parente idoso dependente. A maior dificuldade é quando a dependência ocorre de maneira súbita e não há tempo suficiente para uma organização eficaz da família em torno do idoso hospitalizado. Quando um idoso dependente é internado, as famílias se organizam de diferentes maneiras para acompanhá-lo, de acordo com seus recursos humanos e financeiros disponíveis.

A doença oncológica altera significativamente o cotidiano do familiar/acompanhante da criança no tocante as necessidades biológicas como higiene corporal/banho diário, alimentação e sono.

Quanto às necessidades sociais, os familiares mencionam o auxílio que recebem da família, dos amigos, da igreja e da comunidade, que em algumas vezes ocorre de forma espiritual através de palavras de conforto e demonstração de carinho e em outras vezes com apoio financeiro ou doações de alimentos, roupas, entre outros. Em relação aos aspectos emocionais, destaca-se o sofrimento do familiar/acompanhante quanto ao diagnóstico, tratamento e consequências da doença oncológica.

Quando o idoso é hospitalizado a rotina de sua família é alterada, pois precisa reorganizar-se entre afazeres domésticos, o cuidado dos filhos e o trabalho formal, para assumir a sobrecarga de ter de acompanhar o idoso no hospital. A identificação de alguns fatores estressantes no processo de cuidar de idoso dependente, a partir da perspectiva da família cuidadora, se revelou como uma questão que requer urgentes encaminhamentos, com vista ao aperfeiçoamento da operacionalização de políticas, programas e serviços que levem o sistema público de saúde a prestar uma efetiva e contínua assistência. rentes setores de internação. Foi possível observar a predominância da figura materna como acompanhante, porém os pais também foram incluídos nos estudos. Quatro estudos não mencionaram o grau de parentesco do familiar cuidador. Observou-se que a maioria dos estudos se refere ao familiar cuidador de crianças hospitalizadas.

\section{Discussão}

No que se refere às vivências dos familiares cuidadores frente à situação de hospitalização, são evidentes as mudanças que ocorrem e transformam o cotidiano familiar; envolvendo alteraçóes emocionais de todos os participantes do processo [10]. Quando um indivíduo é acometido por uma 
doença, todo o contexto familiar sofre diante das possibilidades de perda, do desconhecido e do momento incerto [12].

Esta revisão revela a presença de sentimentos variados durante o processo de hospitalização como dor, angústia, tristeza, impotência, medo, desespero, ansiedade e expectativa infinita [11]. Diante de tal situação, nota-se que o padrão de sono do familiar cuidador torna-se irregular devido à preocupação com o estado do paciente, com os ruídos dos equipamentos, punção venosa, administração de medicamentos, entre outros. Além disso, os familiares cuidadores têm sua rotina totalmente alterada e um exemplo está no fato de que muitos tomam banho apenas durante o horário de sono do paciente para que os mesmos não fiquem agitados e sozinhos, conforme evidenciado no estudo citado acima [15]. Cabe ressaltar que segundo a hierarquia das necessidades de Maslow, as necessidades fisiológicas como banho, eliminações, ingestão de água, padrão de sono, encontram-se como primordiais e indispensáveis para manutenção do equilíbrio do organismo. Desta forma o familiar cuidador precisa estar atento quanto a estas questóes.

Um estudo realizado com o objetivo de comparar o estresse de familiares frente à internação domiciliar ou hospitalar apontou que a internaçáo domiciliar está associada a baixos níveis de estresse nos familiares quando comparada aqueles que receberam cuidados hospitalares tradicionais, corroborando os estudos analisados nesta revisão [17].

No estudo de Vieira, Alvarez e Gonçalves [16] foram identificados como fatores que contribuíram para o aumento do estresse dos familiares durante a hospitalização:

A preocupação constante com a manipulação da sonda naso-enteral (SNE) e a realização de curativos ou procedimentos que exigiam habilidades específicas.

Sentimentos como angústia devido à situação de dependência nos cuidadores, pois o ser humano tem como padrấo de saúde a independência na realização das suas atividades diárias.

Sentimento de culpa e frustração em não poder oferecer à pessoa dependente o conforto merecido.

Relaçóes familiares conturbadas devido a problemas financeiros ou sobrecarga de alguns membros no cuidado ao paciente. $\mathrm{O}$ que influencia negativamente na recuperação do mesmo e na organização do cuidado familiar.

Escassez de recursos financeiros, pois são necessários gastos com fraldas, alimentação especial e medicamentos, e estes, quando disponibilizados por órgãos públicos, exigem tempo de espera devido às questôes burocráticas.

A forma da família ver a enfermidade pode ser influenciada por fatores como: o estágio da vida familiar; o papel desempenhado pela pessoa enferma no lar; as implicaçóes que o impacto da doença causa em cada indivíduo; e o modo como ela se organiza durante o período de doença [18].

É importante ressaltar que o aparecimento da doença pode levar a interrupçáo do trabalho/ocupação por parte do familiar cuidador para se dedicar exclusivamente ao paciente adoecido, conforme mencionado em alguns artigos desta revisão [15].

Deste modo, muitos familiares, durante o processo de adoecimento e tratamento, acabam abandonando o emprego e a vida social. Com isso, o familiar cuidador deixa de desfrutar da vida diária devido à falta de tempo livre para descansar e realizar atividades sociais [19].

A família se preocupa com o paciente passando a vivenciar também o medo e a insegurança, resultado da incerteza em relaçáo à conduta e ao tratamento. Como o paciente é o foco do cuidado, as necessidades dos familiares são, muitas vezes, desconsideradas pela equipe de enfermagem.

É de suma importância o planejamento das intervençóes de enfermagem no sentido de apoiar e orientar as famílias para o enfrentamento da nova situação, uma vez que as reaçôes familiares interferem diretamente no processo de aceitaçáo, aprendizado do autocuidado e reabilitaçáo da pessoa hospitalizada [20].

Nota-se nos artigos que o adoecimento de um ente é fator de extrema preocupaçáo para os familiares, pois requer uma nova organização familiar para enfrentar a situaçáo. Desta forma o apoio dos profissionais de saúde é encarado como uma ajuda e facilidade para o cuidado $[12,13]$.

Os resultados do estudo de Silveira e Oliveira [15] enquadram como aspectos facilitadores no processo de hospitalização o auxílio que os familiares cuidadores recebem dos amigos, vizinhos e outros parentes através das visitas. Segundo os relatos dos familiares cuidadores, a força e o apoio destes, durante as visitas, ajudam a minimizar seus medos e angústias, bem como a vivenciar alguns momentos de prazer e de relaxamento.

Foi possível observar que a principal rede de apoio para o enfrentamento das dificuldades, mencionada nos estudos selecionados foi a espiri- 
tualidade. Independente da crença religiosa ficou evidente que a fé foi o apoio buscado com maior frequência durante este momento. $\mathrm{O}$ familiar cuidador utiliza a oração como importante forma ou estratégia para amenizar o sofrimento causado pela hospitalizaçáo. Além disso, ao interagir com outras pessoas o familiar cuidador constrói vínculos que o auxilia a vivenciar as experiências cotidianas de forma menos traumática.

Em um estudo qualitativo com o objetivo de conhecer os sentimentos dos familiares de pacientes internados na Unidade de Terapia Intensiva (UTI), os resultados mostraram que os sentimentos de medo, angústia e desespero poderiam ser minimizados se, durante as visitas, os profissionais de saúde, em especial enfermeiros, oferecessem o acolhimento a esses familiares, preparando-os para a situação de seu familiar internado, escutando-os e esclarecendo dúvidas [11].

Conhecer as formas de apoio vivenciadas pelo familiar cuidador pode favorecer a instrumentalização dos profissionais para um melhor direcionamento de açóes e cuidados destinados à família e ao paciente hospitalizado.

É importante que a equipe de enfermagem se faça presente no processo de cuidar por meio de uma abordagem holística capaz de contemplar o indivíduo e o familiar em seus aspectos multidimensionais [21].

Neste momento de sofrimento e angustia, é imprescindível que haja entre paciente, familiares e equipe de enfermagem uma relação de afeto, respeito e atenção, de modo a potencializar as capacidades e habilidades das pessoas hospitalizadas [22]. Cabe ao enfermeiro, considerar no planejamento de suas açóes, não só a pessoa hospitalizada, mas todos aqueles que se encontram vinculados a ela.

\section{Conclusão}

A partir dos estudos analisados, nota-se que a família está envolvida no processo de hospitalização, sendo necessário que a mesma desenvolva açóes de cuidado, a fim de contribuir para uma melhor recuperaçáo de seu ente.

Foi possível perceber a presença de sentimentos variados, durante o processo de hospitalização como dor, angústia, tristeza, impotência, medo, desespero, ansiedade e expectativa infinita.

Cabe ressaltar que a enfermagem deve estar preparada para atender às necessidades referidas pelos familiares, relacionadas à experiência com a internação, percebendo a família como foco e não como apêndice do cuidado.

Desta forma, torna-se importante o desenvolvimento de capacitaçóes visando a preparação desses profissionais para que possam propiciar o fornecimento adequado de informaçóes, apoio, respeito e atenção tanto aos pacientes quanto aos familiares. Cabe ao enfermeiro, considerar no planejamento de suas açóes não só a pessoa hospitalizada, mas todos aqueles que se encontram vinculados a ela.

\section{Referências}

1. Pinto J, Ribeiro C, Silva C. Procurando manter o equilíbrio para atender as demandas e cuidar da criança hospitalizada: a experiência da família. Rev Latinoam Enferm 2005;13:974-81.

2. Souza JL, Gomes GC, Barros EJL. O cuidado à pessoa portadora de estomia: o papel do familiar cuidador. Rev Enferm UERJ 2009;17:550-55.

3. Pena SB, Diogo MJD. Fatores que favorecem a participação do acompanhante no cuidado do idoso hospitalizado. Rev Latinoam Enferm 2005;13:663-9.

4. Sousa L, Gomes G, Santos C. Percepçóes da equipe de enfermagem acerca da importância da presença do familiar/acompanhante no hospital. Rev Enferm UERJ 2009;3:394-9.

5. Sousa LD, Gomes GC, Silva MRS, Santos CP, Silva BT. A família na unidade de pediatria: percepçóes da equipe de enfermagem acerca da dimensão cuidadora. Ciencia y Enfermeria 2011;17:87-95.

6. Oliveira GS, Bavaresco M, Filipini CB, Rosado SR, Dázio EMR, Fava SMCL. Vivências do cuidador familiar de uma pessoa com estomia intestinal por câncer colorretal. Rev RENE 2014; 15:108-15.

7. Mendes KDS, Silveira RCP, Galvão CM. Revisão integrativa: método de pesquisa para a incorporação de evidências na saúde e na enfermagem. Texto Contexto Enferm 2008;17:758-64.

8. Ursi ES. Prevenção de lesões de pele no perioperatório: revisão integrativa da literatura [Dissertação]. Ribeirão Preto: Escola de Enfermagem Ribeirão Preto, Universidade de São Paulo; 2005.

9. Melnyk BM, Fineout-Overholt E. Evidence based practice in nursing $\&$ healthcare: a guide to best practice. 2nd ed. Philadelphia: Wolters Kluwer Health/ Lippincot Williams \& Wilkins; 2011.

10. Santos AMR, Amorim NMA, Braga CH, Lima FDM, Macedo EMA, Lima CF. Vivências de familiares de crianças internadas em um Serviço de Pronto-Socorro. Rev Esc Enferm 2011;45:473-9.

11. Frizon G, Nascimento ERP, Bertoncello KCG, Martins JJ. Familiares na sala de espera de uma unidade de terapia intensiva: sentimentos revelados. Rev Gaúcha Enferm 2011;32:72-8.

12. Mello RM, Schneider JF. A família e a internação psiquiátrica em hospital geral. Rev Gaúcha Enferm 2011;32:226-33. 
13. Gomes CC, Pintanel AC, Strasburg AC, Erdmann AL. O apoio social ao familiar cuidador durante a internação hospitalar da criança. Rev Enferm UERJ 2011;19:64-9.

14. Vieira GB, Alvarez AM, Girondi JBR. O estresse do familiar acompanhante de idosos dependentes no processo de hospitalização. Rev Eletrônica Enferm 2011;13:78-9.

15. Silveira RA, Oliveira ICS. O cotidiano do familiar/ acompanhante junto da criança com doença oncológica durante a hospitalização. Rev RENE 2011;12:532-9.

16. Vieira GB, Alvarez AM, Gonçalves LTI. A enfermagem diante dos estressores de familiares acompanhantes de idosos dependentes no processo de hospitalização e de alta. Ciênc Cuidado Saúde 2009;8:645-51.

17. Leff B, Burton L, Mader SL, Naughton B, Burl J, Koehn D, et al. Comparison of stress experienced by family members of patients treated in hospital at home with that of those receiving traditional acute hospital care. J Am Geriatr Soc 2008;56:117-23.

18. Larrobla C, Botega NJ. Hospitais gerais filantrópicos: novo espaço para a internaçáo psiquiátrica. Rev Saúde Pública 2006; 40:1042-8.

19. Sales CA, Matos PCB, Mendonça DPR, Marcon SS. Cuidar de um familiar com câncer: o impacto no cotidiano de vida do cuidador. Rev Eletrônica Enferm 2010;12:616-21.

20. Crema E, Silva R. Estomas: uma abordagem interdisciplinar. Uberaba: Pinti; 1997.

21. Razera APR, Braga EM. A importância da comunicação durante o período de recuperaçáo pós-operatória. Rev Esc Enferm USP 2011;45:632-7.

22. Fontes CAS, Alvim NAT. Human relations in nursing care towards cancer patients submitted to antineoplastic chemotherapy. Acta Paul Enferm 2008;21:77-83. 